\title{
AVI as a mechanical tool for studying dynamic and static beam structures
}

\author{
François Demoures, Tudor S. Ratiu, Yves Weinand \& Julien Nembrini \\ Ecole Polytechnique Fédérale de Lausanne, Switzerland
}

\begin{abstract}
In this paper we study the dynamic behavior of an isotropic KirchhoffLove beam and investigate the equilibrium position of an Euler-Bernoulli beam. Using the discrete Euler-Lagrange and Lagrange-d'Alembert principles, we simulate the behavior by means of variational integrators and, in particular, AVIs (Asynchronous Variational Integrators). We place special emphasis on the geometric structure underlying stress resultant beam models and propose B-spline shape functions for AVI-method.
\end{abstract}

\section{INTRODUCTION}

Discrete variational mechanics and associated numerical integrators have seen a major development in recent years. There has been a growing realization that stability of numerical methods can be improved for certain systems by algorithms that are compatible with variational and geometric structures, such as preservation of the symplectic form on phase space and of the momentum maps arising from the symmetries of the system. Discrete mechanics has been developed as a result of the interplay of classical theoretical mechanics, numerical analysis, and computer science. It has become increasingly important in concrete applications. Remarkably, to our knowledge, there is no major application of these discrete mechanics techniques to civil engineering.

The aim of this note is to apply structure preserving algorithms to concrete problems in construction, like thin-shells with irregular surfaces. The major future objective is the search and development of a practical tool to study these irregular surfaces. Here we consider a three dimensional isotropic Kirchhoff-Love beam model as a special kind of shell having a narrow width. Our goal is to provide variational integrators associated with mathematical models of beams and to carry out a dynamic and static two-dimensional simulation.

\section{NOTATION AND DEFINITIONS}

Denote by $\mathcal{B}$ the material reference configuration of the shell, assumed to be a smooth closed two-dimensional submanifold of $\mathbb{R}^{3}$. A deformed state of the shell, that is, a configuration, is given by an embedding $\phi: \mathcal{B} \rightarrow \mathcal{S}=\mathbb{R}^{3}$, where $\mathcal{S}$ is physical space. Let $\mathcal{C}$ be the set of all such configurations. A motion of $\mathcal{B}$ is a curve $t \in \mathbb{R} \mapsto \phi_{t}=\phi(t, \cdot) \in \mathcal{C}$. If $\left\{\theta^{\alpha}\right\}, \alpha=1,2$, is a coordinate system on $\mathcal{B}$, the configuration $\phi$ is written as

$$
\left(\theta^{1}, \theta^{2}\right) \in \mathcal{B} \stackrel{\phi}{\longmapsto}\left(z^{1}\left(\theta^{1}, \theta^{2}\right), z^{2}\left(\theta^{1}, \theta^{2}\right), z^{3}\left(\theta^{1}, \theta^{2}\right)\right) \in \mathbb{R}^{3},
$$

where $\left\{z^{j}\left(\theta^{1}, \theta^{2}\right)\right\}$ are the Euclidean coordinates of the spatial point $\mathbf{x}=\phi(\mathbf{X})$, where the material point $\mathbf{X} \in \mathcal{B}$ has coordinates $\left(\theta^{1}, \theta^{2}\right)$. 
In the standard spatial orthonormal basis $\left\{\widehat{\mathbf{i}}_{j}\right\}, j=1,2,3$, of $\mathcal{S}=\mathbb{R}^{3}$, the basis vectors of the tangent space $T_{\mathbf{x}}(\phi(\mathcal{B}))$ to the current configuration $\phi(\mathcal{B})$ are given by

$$
\mathbf{e}_{\alpha}:=\frac{\partial z^{j}}{\partial \theta^{\alpha}} \widehat{\mathbf{i}}_{j}, \quad \alpha=1,2, \quad j=1,2,3 .
$$

Let $\left\{Z^{j}\left(\theta^{1}, \theta^{2}\right) \mid j=1,2,3\right\}$ be the Euclidean coordinates of the material point $\mathbf{X} \in \mathcal{B} \subset$ $\mathbb{R}^{3}$ and let $\left\{\mathbf{E}_{\alpha} \mid \alpha=1,2\right\}$ be the basis of the tangent space $T_{\mathbf{X}} \mathcal{B}$ to the material reference configuration associated to the coordinate system $\left\{\theta^{1}, \theta^{2}\right\}$. According to standard Kirchhoff-Love assumptions, we take the reference shell director ${ }^{1} \mathbf{T}$ to equal the third basis vector

$$
\mathbf{E}_{3}=\frac{\mathbf{E}_{1} \times \mathbf{E}_{2}}{\left|\mathbf{E}_{1} \times \mathbf{E}_{2}\right|} \perp T_{\mathbf{X}} \mathcal{B}
$$

In this work we consider the simplest properly invariant isotropic ${ }^{2}$ constitutive relations for the effective membrane and shear stress resultants. So we define the unit normal

$$
\mathbf{e}_{3}=\frac{\mathbf{e}_{1} \times \mathbf{e}_{2}}{\left|\mathbf{e}_{1} \times \mathbf{e}_{2}\right|}
$$

to the deformed surface $\phi(\mathcal{B})$ to be the deformed shell director $\mathbf{t}$.

Denote by $\langle\cdot, \cdot\rangle_{\mathbf{x}}$ the standard inner product in $\mathbb{R}^{3}$ for vectors based at $\mathbf{x} \in \mathcal{S}=\mathbb{R}^{3}$ and by $\langle\cdot, \cdot\rangle_{\mathbf{X}}$ the standard inner product in $\mathbb{R}^{3}$ for vectors based at $\mathbf{X} \in \mathcal{B}$. The components $g_{\alpha \beta}$ of the metric tensor on $\phi(\mathcal{B})$ (obtained by pulling back by the inclusion map the inner product $\langle\cdot, \cdot\rangle_{\mathbf{x}}$ on $\mathbb{R}^{3}$ to $\left.\phi(\mathcal{B})\right)$ are defined by $g_{\alpha \beta}(\mathbf{x}):=\left\langle\mathbf{e}_{\alpha}, \mathbf{e}_{\beta}\right\rangle_{\mathbf{x}}$. Similarly define the components $G_{\alpha \beta}$ of the metric on $\mathcal{B}$ by $G_{\alpha \beta}(\mathbf{X}):=\left\langle\mathbf{E}_{\alpha}, \mathbf{E}_{\beta}\right\rangle_{\mathbf{X}}$. Let $\left[G^{\alpha \beta}\right]:=\left[G_{\alpha \beta}\right]^{-1}$ and $\left[g^{\alpha \beta}\right]:=\left[g_{\alpha \beta}\right]^{-1}$.

\section{DISCRETE VARIATIONAL MECHANICS}

Let $\phi(\mathcal{B}) \times \phi(\mathcal{B})$ be the discrete configuration space associated to the deformed surface $\phi(\mathcal{B})$ and define the discrete path space by $\mathcal{C}_{d}(\phi(\mathcal{B})):=\left\{\mathbf{x}_{d}=\left\{\mathbf{x}_{k}\right\}_{k=0}^{N} \mid \mathbf{x}_{k} \in \phi(\mathcal{B}), \mathbf{x}_{k}=\right.$ $\left.\mathbf{x}_{d}\left(t_{k}\right), t_{k}=k h, t_{k} \in[0, T]\right\} ; h$ is the time step. A discrete path $\mathbf{x}_{d} \in \mathcal{C}_{d}$ is said to be a solution of the discrete Euler-Lagrange equations if

$$
D_{2} L_{d}\left(\mathbf{x}_{k-1}, \mathbf{x}_{k}\right)+D_{1} L_{d}\left(\mathbf{x}_{k}, \mathbf{x}_{k+1}\right)=0, \quad \text { for all } k=1, \ldots, N-1,
$$

where $L_{d}: \phi(\mathcal{B}) \times \phi(\mathcal{B}) \rightarrow \mathbb{R}$ is a discrete Lagrangian of order $r$, that is, it satisfies

$$
L_{d}\left(\mathbf{x}_{k}, \mathbf{x}_{k+1}, \Delta t\right)=\int_{t_{k}}^{t_{k+1}} L(\mathbf{x}, \dot{\mathbf{x}}) d t+\mathcal{O}(\Delta t)^{r+1}
$$

where $L$ is the Lagrangian of the continuous systems and $\mathbf{x}(t)$ is the solution of the EulerLagrange equations satisfying $\mathbf{x}\left(t_{k}\right)=\mathbf{x}_{k}$ and $\mathbf{x}\left(t_{k+1}\right)=\mathbf{x}_{k+1}$.

\section{AVI AS MECHANICAL TOOL FOR BEAM DYNAMICS}

\subsection{Thin-shell with uniform and small width}

In this section we consider a thin shell with uniform small width satisfying the KrichhoffLove hypotheses. With these assumptions the shell can be regarded as beam.

\footnotetext{
${ }^{1}$ We assume the existence of a traction vector $\mathbf{t}$ for the motion of $\mathcal{B}$ in $\mathcal{S}$.

${ }^{2}$ Isotropic at a point means that all orientation preserving rotations about that point are material symmetries.
} 
The strain mesures relative to the dual spatial surface basis are given by ([4], p. 287):

$$
\begin{aligned}
\epsilon_{i j} & :=\frac{1}{2}\left(\left\langle\mathbf{e}_{i}, \mathbf{e}_{j}\right\rangle-\left\langle\mathbf{E}_{i}, \mathbf{E}_{j}\right\rangle\right) \\
\rho_{\alpha \beta} & :=\left\langle\frac{\partial \mathbf{E}_{\alpha}}{\partial \theta^{\beta}}, \mathbf{E}_{3}\right\rangle-\left\langle\frac{\partial \mathbf{e}_{\alpha}}{\partial \theta^{\beta}}, \mathbf{e}_{3}\right\rangle .
\end{aligned}
$$

For the simplest properly invariant isotropic constitutive relations we postulate the existence of a stored energy function of the displacement field $\mathbf{u}$ of the form ([3], p. 2044)

$$
W(\mathbf{u})=\frac{1}{2}\left(\frac{E h}{1-\nu^{2}}\right) H^{\alpha \beta \gamma \delta} \epsilon_{\alpha \beta} \epsilon_{\gamma \delta}+\frac{1}{2}\left(\frac{E h^{3}}{12\left(1-\nu^{2}\right)}\right) H^{\alpha \beta \gamma \delta} \rho_{\alpha \beta} \rho_{\gamma \delta}
$$

where $E$ is Young's modulus, $\nu$ is Poisson's ratio, $h$ is the thickness of the shell, and

$$
H^{\alpha \beta \gamma \delta}=\nu G^{\alpha \beta} G^{\gamma \delta}+\frac{1}{2}(1-\nu)\left(G^{\alpha \gamma} G^{\beta \delta}+G^{\alpha \delta} G^{\beta \gamma}\right) .
$$

To ensure that the bending energy is finite we use B-splines ${ }^{3}$. The general form of quadratic uniform B-splines is:

$$
\mathbf{x}_{a}(u)=\frac{1}{2}\left(u^{2}, u, 1\right)\left(\begin{array}{rrr}
1 & -2 & 1 \\
-2 & 2 & 0 \\
1 & 1 & 0
\end{array}\right)\left(\mathbf{x}_{a-1}, \mathbf{x}_{a}, \mathbf{x}_{a+1}\right)^{T}, \quad \text { with } \quad 0 \leqslant u \leqslant 1,
$$

where $a$ is a node of the triangulation $\mathcal{T}$ of the beam in the reference configuration $\mathcal{B}$ and $a-1, a+1$ are the neighboring nodes of $a$. For a 1-simplex $K \in \mathcal{T}$ associated to the beam, the local Lagrangian has the form

$$
L_{K}\left(\mathbf{x}_{K}, \dot{\mathbf{x}}_{K}, t\right)=T_{K}\left(\dot{\mathbf{x}}_{K}\right)-V_{K}\left(\mathbf{x}_{K}, t\right),
$$

$\mathbf{x}_{K}$ is the vector of positions of all nodes in the element $K, V_{K}\left(\mathbf{x}_{K}, t\right)$ is the elemental potential energy, and

$$
T_{K}\left(\dot{\mathbf{x}}_{K}\right)=\frac{1}{2} \dot{\mathbf{x}}_{K}^{t} \mathbf{m}_{K} \dot{\mathbf{x}}_{K}
$$

where $\mathbf{m}_{K}$ is the mass matrix element (symmetric positive definite).

A particular choice of discrete Lagrangian, resulting in explicit integrators of the centraldifference type, is given by

$$
L_{K}^{j}=\int_{t_{K}^{j}}^{t_{K}^{j+1}} T_{K}\left(\dot{\mathbf{x}}_{K}(t)\right) d t-\left(t_{K}^{j+1}-t_{K}^{j}\right) V_{K}\left(\mathbf{x}_{K}^{j+1}, t_{K}^{j+1}\right)
$$

where $L_{K}^{j}$ is defined on the interval $\left[t_{K}^{j}, t_{K}^{j+1}\right]$ for $t_{K}^{j}=j \Delta t_{K}$ (the $j$ th time step for the element $K$ ). To guarantee energy conservation it is necessary to ensure that a valid time step for each element is always determined for each $t_{j}$. As noted in [1], p. 199, if we fix the time step $\Delta t_{K}$ for each simplex $K$, the total energy of the system oscillates around a constant value without growth or decay.

The corresponding discrete action is

$$
\begin{aligned}
S_{d} & =\sum_{K \in \mathcal{T}} \sum_{1 \leqslant j<N_{K}} L_{K}^{j} \\
& \approx \sum_{a} \sum_{i=0}^{N_{a}-1} \frac{1}{2} m_{a}\left(t_{a}^{i+1}-t_{a}^{i}\right)\left\|\frac{\mathbf{x}_{a}^{i+1}-\mathbf{x}_{a+1}^{i}}{t_{a}^{i+1}-t_{a}^{i}}\right\|^{2}-\sum_{K} \sum_{j=0}^{N_{K}-1}\left(t_{K}^{j+1}-t_{K}^{j}\right) V_{K}\left(\mathbf{x}_{K}^{j+1}\right),
\end{aligned}
$$

\footnotetext{
${ }^{3}$ In $\mathbb{R}^{3}$, if we want smallest element in the mesh, the subdivision of the surface obtained by loop or Catmull-Clark subdivisions are guaranted to be $H^{2}$.
} 
where $\mathbf{x}_{a}^{i}$ is the position of the node $a$ at time $t_{a}^{i}$. The position and the time are dependent on two 1-simplices, one on each side.

The discrete version of Hamilton's principle states that the discrete trajectory having prescribed initial and final endpoints renders the discrete action stationary with respect to admissible variations of the nodal coordinates $\mathbf{x}_{a}$. This leads to the discrete EulerLagrange equations $D_{a}^{i} S_{d}=0$ for all $a \in \mathcal{T}$ such that $t_{\text {initial }}<t_{a}^{i}<t_{\text {final }}$ and $a \in \mathcal{T} \backslash \partial_{d} \mathcal{B}$, where $\partial_{d} \mathcal{B}$ is the discretization of the boundary of the reference configuration $\mathcal{B}$.

Thus, the element $K$ accumulates and memorizes the impulses over the time interval $\left(t_{K}^{j-1}, t_{K}^{j}\right)$. At the end of the interval, the element releases its memorized impulses by imparting percussions on its nodes.

\subsection{Simulations}

The mechanical model on which the simulation results presented here are based does not have an analytical solution. Thus, to calibrate such results requires an appropriate experimental setup able to display quantifiable dynamic and static behavior. This is not presented in this paper and left for a future publication. In the following section, the same approach is applied to the Bernoulli beam problem presenting in this case an exact analytical solution.

Figure 4.2 displays the behavior of several beams with increasing stiffness. These results were obtained using a module of elasticity $E$ ranging from $E=11$ to $E=$ $11000, \nu=0.3$, with a beam of length $L=2 \mathrm{~m}$, width $h=1 \mathrm{~mm}$, and density $\rho=400 \mathrm{~kg} / \mathrm{m}^{3}$. The time of the experiment is one second in each case.

In all experiments, the value of the time step for each element is computed as $\Delta t=f \frac{h}{c}$ where $f=\frac{1}{100}$ (or close to it), and $h$ is the radius of the largest ball contained in the element.

The time steps in seconds for each 1-simplex, from left to right, are:

$$
\text { 0.0046, 0.0066, 0.0098, 0.0120, 0.0139, 0.0148, 0.0139, 0.0120, 0.0098, 0.0066. }
$$

If $N$ is the number of nodes on the beam, define the position $n_{a}$ of the node $a$ at time $t=0$ on the beam by using the rules

$$
\mathbf{X}_{a}=2^{S-1}\left(\frac{n_{a}-1}{N-1}\right)^{S} L, \quad \mathbf{X}_{a}=\left(1-2^{S-1}\left(\frac{N-n_{a}}{N-1}\right)^{S}\right) L
$$

the first for the first $\frac{N}{2}$ nodes and the second for the last $\frac{N}{2}$ nodes.

In practice, even when the step time is not adapted to each step, the total energy of the system oscillates around a constant value for very long times without growth or decay. Energy behaviour is excelent locally as well as globally.

\section{AVI FOR PLANAR EULER-BERNOULLI BEAM STATICS}

To get an equilibrium position in a static problem we use a system with dissipation.

\subsection{Discrete Lagrange-d'Alembert principle}

We consider a non-conservative system with dissipation. The discrete version of the Lagrange-d'Alembert principle states that the discrete trajectory $\left\{q_{k}\right\}$, with prescribed initial and final endpoints, is a solution of the equation

$$
\delta \sum_{k=0}^{N} L_{d}\left(q_{k}, q_{k+1}\right)+\sum_{k=0}^{N}\left[F_{d}^{-}\left(q_{k}, q_{k+1}\right) \delta q_{k}+F_{d}^{+}\left(q_{k}, q_{k+1}\right) \delta q_{k+1}\right]=0
$$



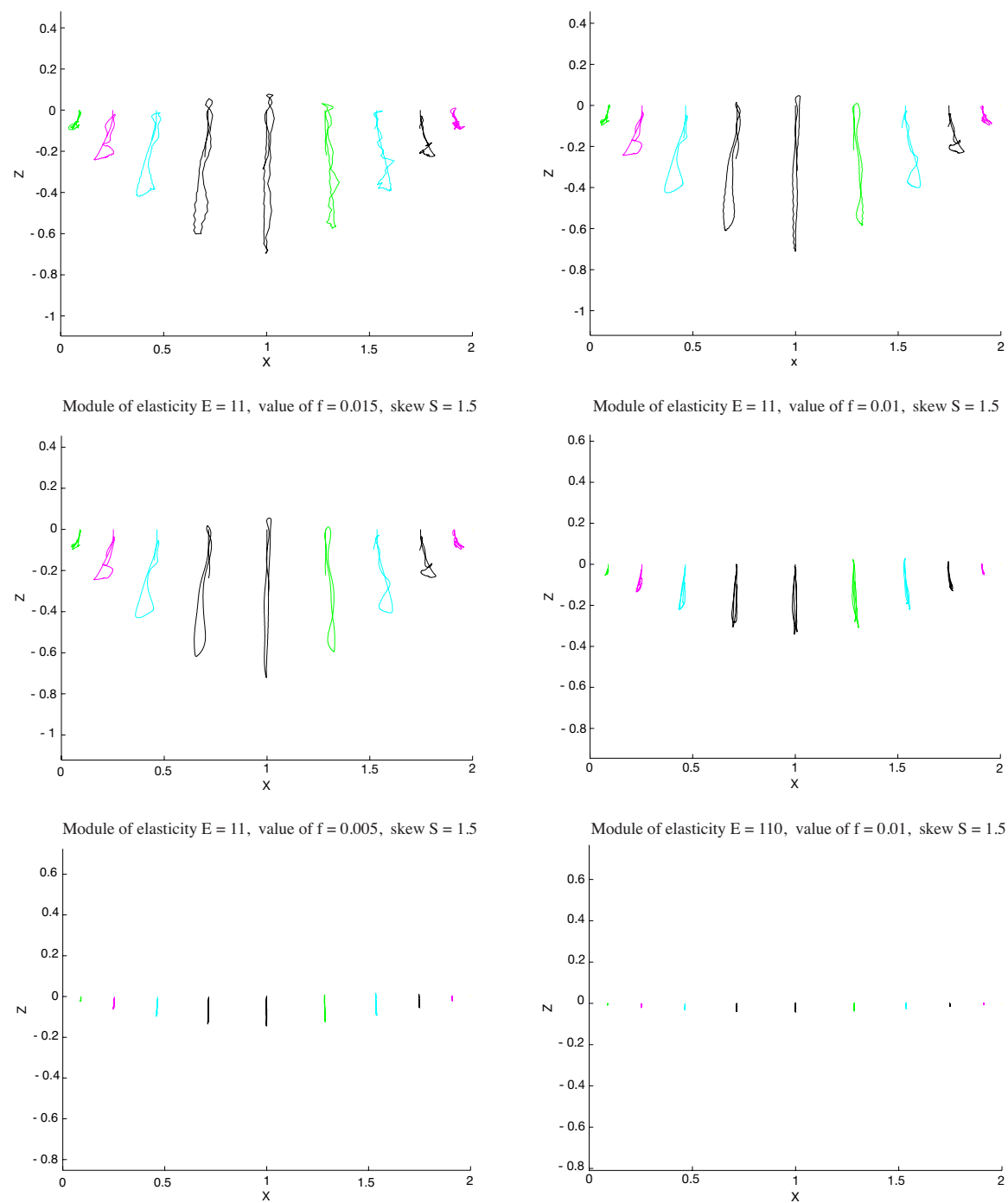

Module of elasticity $\mathrm{E}=1100$, value of $\mathrm{f}=0.01$, skew $\mathrm{S}=1.5$

Module of elasticity $E=11000$, value of $f=0.01$, skew $\mathrm{S}=1.5$

Figure 1: Simulation trajectories of the thin-shell model for different material stiffness

where $F_{d}^{-}$and $F_{d}^{+}$are left and right discrete forces. The associated forced discrete Euler-Lagrange equations are hence

$$
D_{2} L_{d}\left(q_{k}, q_{k+1}\right)+D_{1} L_{d}\left(q_{k+1}, q_{k+2}\right)+F_{d}^{+}\left(q_{k}, q_{k+1}\right)+F_{d}^{-}\left(q_{k+1}, q_{k+2}\right)=0 .
$$

In our case we use the discrete force

$$
F_{d}^{-}\left(q_{k}, q_{k+1}\right)=0, \quad F_{d}^{+}\left(q_{k}, q_{k+1}\right)=-\lambda \frac{\left(q_{k+1}-q_{k}\right)}{\left(t_{k+1}-t_{k}\right)}, \quad \lambda \in \mathbb{R} .
$$

\subsection{Euler-Bernoulli planar beam}

For the Euler-Bernoulli beam the assumptions of infinitesimal deformations are applied and the material is assumed to be hyperelastic and isotropic. Let $\mathcal{B}$ be the reference configuration of the beam, $\mathbf{G}$ the metric on $\mathcal{B}$, and $\mathrm{g}$ the metric on $\mathcal{S}$. Assume that the configuration $\phi$ is of the form

$$
\phi: \mathcal{B} \rightarrow \phi(\mathcal{B}) \subset \mathcal{S}, \quad \mathbf{X} \mapsto \mathbf{x}=\phi(\mathbf{X})=\mathbf{X}+\mathbf{u}(\mathbf{X}),
$$


where $\mathbf{u}(\mathbf{X})$ is the displacement.

The cross-section of the undeformed beam is determined by unit length vectors $\mathbf{i}_{1}$ and $\mathbf{i}_{2}$, such that $\left\{\mathbf{i}_{I}\right\}_{I=1,2,3}$ is an orthonormal basis chosen to be the standard basis of $\mathcal{S}$. The cross-section $\mathcal{A} \subset \mathbb{R}^{2}$ of the beam is an open set with compact closure. Points in $\mathcal{A}$ are written as $\mathbf{s}=\xi^{1} \mathbf{i}_{1}+\xi^{2} \mathbf{i}_{2}$.

Consider the orthogonal transformation :

$$
\mathbb{R} \rightarrow \mathrm{SO}(3), \quad \xi^{3} \mapsto \boldsymbol{\Lambda}\left(\xi^{3}\right),
$$

such that $\mathbf{d}_{I}\left(\xi^{3}\right)=\boldsymbol{\Lambda}\left(\xi^{3}\right) \mathbf{i}_{I}$, where $\left\{\mathbf{d}_{I}\right\}$ the standard orthonormal basis of $\mathcal{S}=\mathbb{R}^{3}$ after deformation. The cross-section is now determined by $\mathbf{d}_{1}\left(\xi^{3}\right)$ and $\mathbf{d}_{2}\left(\xi^{3}\right)$. The spatial representation of the beam is:

$$
\mathbf{x}=\phi(\mathbf{X})=\varphi_{0}\left(\xi^{3}\right)+\sum_{\alpha=1}^{2} \xi^{\alpha} \mathbf{d}_{\alpha}\left(\xi^{3}\right),
$$

where $\xi^{3} \in I \subset \mathbb{R} \mapsto \varphi_{0}\left(\xi^{3}\right) \in \mathbb{R}^{3}$ is the line of centroids.

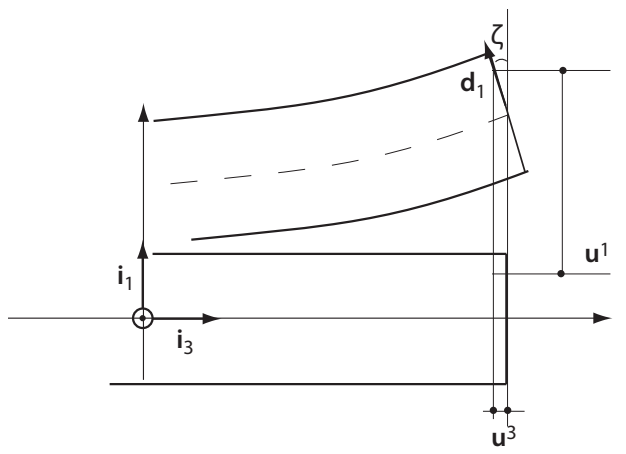

Figure 2: Deflection of the Bernoulli beam

In this work we assume throughout than $\left\{\mathbf{i}_{i}\right\}$ is the orthonormal basis of $\mathbb{R}^{3}, \mathbf{G}=\mathbf{I}$, and that the thickness and the width of the beam are uniform.

Let $\xi^{1} \mathbf{d}_{1}\left(\xi^{3}\right)+\xi^{2} \mathbf{d}_{2}\left(\xi^{3}\right)$ denote the position of a point in the cross-section, which remains planar and normal to the line of centroids upon bending the Bernoulli beam. The set of cross-sections has a longitudinal plane of symmetry. Also, for small deformations, with $\zeta$ the slope of the cross-section, we take the displacement $\mathbf{u}(\mathbf{X})$ to have the form

$$
\begin{aligned}
& u^{1}\left(\xi^{1}, \xi^{2}, \xi^{3}\right)=u^{1}\left(\xi^{3}\right) \\
& u^{2}\left(\xi^{1}, \xi^{2}, \xi^{3}\right)=\text { const } \\
& u^{3}\left(\xi^{1}, \xi^{2}, \xi^{3}\right) \approx-\xi^{1} \zeta=-\xi^{1} \frac{\partial u^{1}\left(\xi^{3}\right)}{\partial \xi^{3}}
\end{aligned}
$$

The local internal energy $W(\mathbf{E})$ depends only on the strain tensor

$$
\mathbf{E}=\frac{1}{2}\left(\nabla \mathbf{u}(\mathbf{X})+(\nabla \mathbf{u}(\mathbf{X}))^{\boldsymbol{\top}}\right)
$$

since we consider a linear constitutive law with small deformations. The bending energy function of the displacement field $\mathbf{u}_{K}$ for a beam element $K$ of length $l$ is

$$
W\left(\mathbf{u}_{K}\right)=\frac{1}{2} \int_{0}^{1} l E I\left(\frac{\partial^{2} u^{1}}{\partial \xi^{3} \partial \xi^{3}}\right)^{2} d \xi^{1},
$$


where $E$ is Young's modulus and $I$ is the second moment of the cross section. As previously, we use quadratic uniform B-splines for each beam element $K$

$$
\mathbf{u}_{K}(w)=\frac{1}{6}\left(w^{2}, w, 1\right)\left(\begin{array}{rrr}
1 & -2 & 1 \\
-2 & 2 & 0 \\
1 & 1 & 0
\end{array}\right)\left(\begin{array}{c}
\mathbf{u}_{a} \\
\mathbf{u}_{a+1} \\
\mathbf{u}_{a+2}
\end{array}\right)=\sum_{i=0}^{2} N^{i}(w) \mathbf{u}_{a+i}
$$

where $w \in[0,1], a, a+1, a+2$ are control points, and $\left\{N^{i}\right\}_{i=0,1,2}$ are given by

$$
N^{0}(w)=\frac{1}{2}\left(w^{2}-2 w+1\right), \quad N^{1}(w)=\frac{1}{2}\left(-2 w^{2}+2 w+1\right), \quad N^{2}(w)=\frac{w^{2}}{2} .
$$

The internal energy $W_{K}$ has the expression

$$
W_{K}\left(\mathbf{u}_{K}, t\right)=\frac{1}{2} \int_{0}^{1} l E I \mathbf{u}^{T} \mathbf{B}_{1}^{T} \mathbf{B}_{1} \mathbf{u} d w
$$

where

$$
\mathbf{B}_{1}=\frac{1}{l^{2}}(1,-2,1)
$$

We use Hermitian cubic shape functions as in the finite element method:

$$
\mathbf{u}_{K}(w)=\mathbf{N u}=\left(N_{u_{a}^{2}}(w), N_{\alpha_{a}}(w), N_{u_{a+1}^{2}}(w), N_{\alpha_{a+1}}(w)\right)\left(u_{a}^{2}, \alpha_{a}, u_{a+1}^{2}, \alpha_{a+1}\right)^{\top},
$$

where $w \in[-1,1], a, a+1$ are the extreme nodes of the beam element $K$ and $\mathbf{N}=\left\{N_{u_{a}^{2}}(w), N_{\alpha_{a}}(w), N_{u_{a+1}^{2}}(w), N_{\alpha_{a+1}}(w)\right\}$ is given by

$$
\left\{\begin{array}{l}
N_{u_{a}^{2}}(w)=1 / 4(1-w)^{2}(w+2) \\
N_{\alpha_{a}}(w)=l / 8(1-w)^{2}(w+1) \\
N_{u_{a+1}^{2}}(w)=1 / 4(2-w)(w+1)^{2} \\
N_{\alpha_{a+1}}(w)=-l / 8(1-w)(w+1)^{2}
\end{array}\right.
$$

The internal energy $W_{K}$ is

$$
W_{K}\left(\mathbf{u}_{K}, t\right)=\frac{1}{4} \int_{-1}^{1} l E I \mathbf{u}^{T} \mathbf{B}_{2}^{T} \mathbf{B}_{2} \mathbf{u} d w .
$$

where

$$
\mathbf{B}_{2}=\left(\frac{6 w}{l^{2}}, \frac{3 w-1}{l},-\frac{6 w}{l^{2}}, \frac{3 w+1}{l}\right)
$$

\subsection{Simulation}

Experimental results were obtained using a module of elasticity $E=11.10^{8}$ with a beam of length $L=1 \mathrm{~m}$, width $h=2 \mathrm{~mm}$, depth $=1 \mathrm{~mm}$, and density $\rho=400 \mathrm{~kg} / \mathrm{m}^{3}$. During the numerical experiments we tested end-fixed, hybrid (end-fixed, simply supported), cantilever, simply supported, and intermediate load (end-fixed) situations. Experimental results are associated with true analytical solutions on the same plot, where $s=1$ means uniform mesh, $k$ clamped coefficient, and $D$ the number of elements in the mesh (see Figure 5.3). 

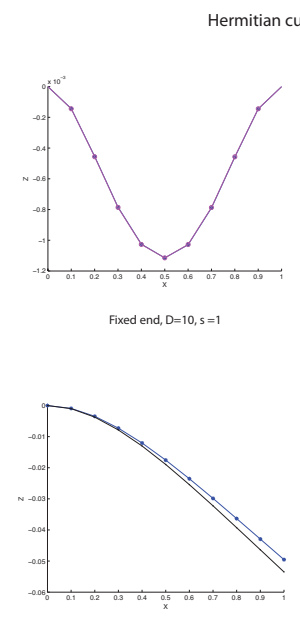

Canteliver, $\mathrm{D}=10,5=1$
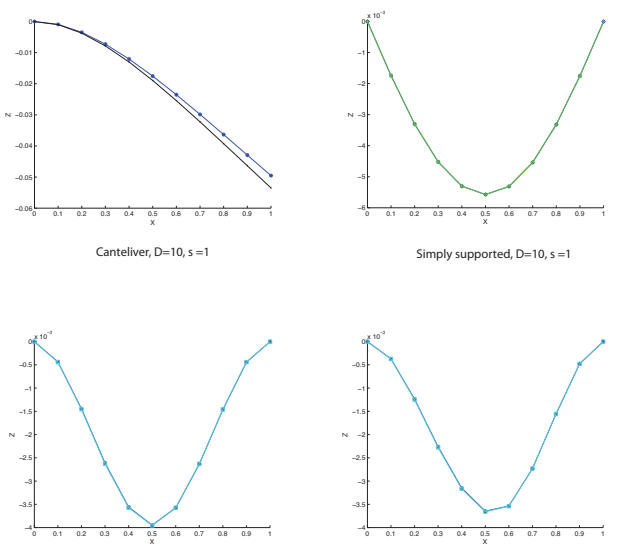

Simply supported, $D=10, s=1$
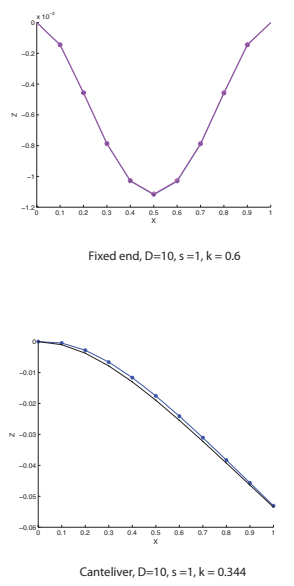

Fixed end, $\mathrm{D}=10, \mathrm{~s}=1, \mathrm{k}=0.6$

Quadratic B-spline
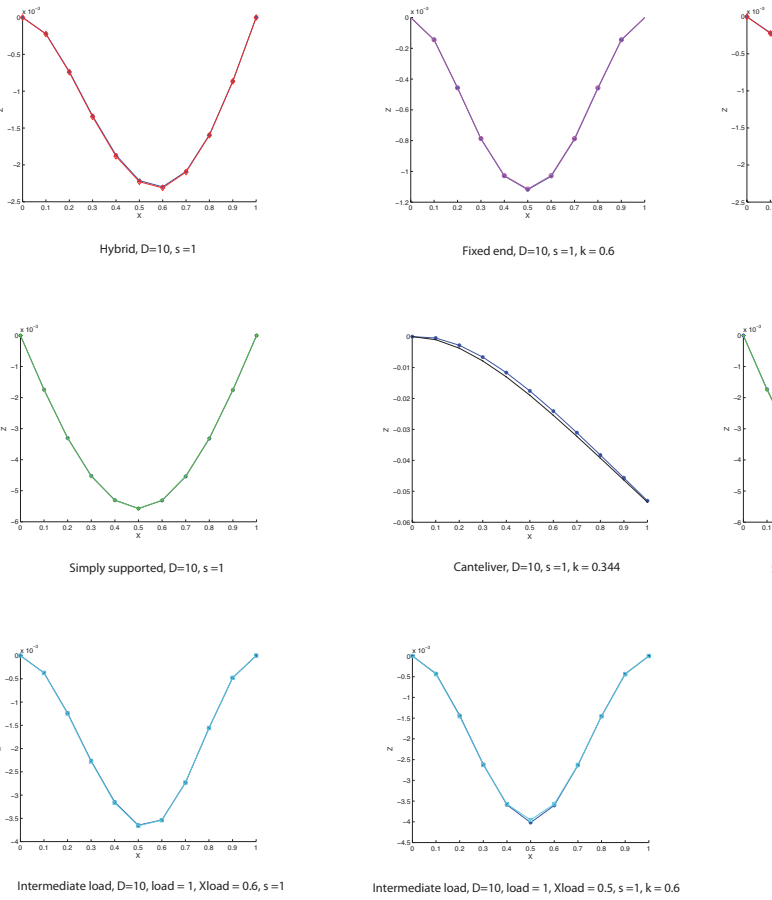
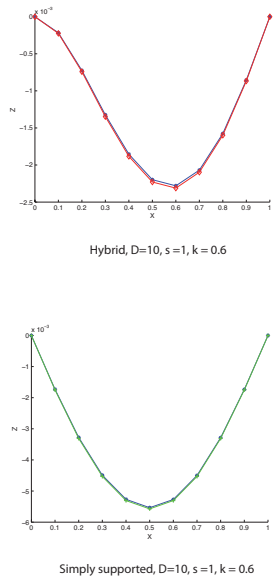

Figure 3: Simulation results of the Bernoulli model using different boundary conditions

\section{REFERENCES}

[1] Lew, A., Marsden, J. E., Ortiz, M., West, M. Variational time integrators. Internat. J. Numer. Methods Engrg., 60(1) (2004), 153-212.

[2] Marsden, J. E., West, M. Discrete mechanics and variational integrators. Acta Numer., 10 (2001), $357-514$.

[3] Cirak, F., Ortiz, M., and Schröder, P. Subdivision surfaces: a new paradigm for thin-shell finiteelement analysis. Int. J. Numer. Meth. Engng., 47 (2000), 2039-2072.

[4] Simo, J. C., Fox, D. D. On a stress resultant geometrically exact shell model. I. Formulation and optimal parametrization. Comput. Methods Appl. Mech. Engrg., 72(3) (1989), 267-304. 